\title{
Convenient formulae for some integrals in perturbation theory
}

\author{
D. Henderson* \\ Department of Chemistry and Biochemistry, Brigham Young University, Provo UT 84602
}

Received March 1, 2010, in final form March 10, 2010

\begin{abstract}
The free energy and pressure of a fluid, as given by perturbation theory, involve integrals of the hard sphere correlation functions and their density derivatives. In most applications a straightforward procedure would be to obtain these integrals, possibly numerically, using the formulae and computer codes for the hard sphere correlation functions, given previously [Mol. Phys., 2007, 106, 2; Condens. Matter Phys., 2009, 12, 127], followed by numerical differentiation with respect to the density and a possible compounding of errors. More sophisticated methods are given in this paper, which is the second in a planned trilogy, drawn from the author's lecture notes. Three representative model fluids are considered. They are the square-well fluid, the Yukawa fluid, and the Lennard-Jones fluid. Each model fluid is popular for theoretical and engineering calculations and can represent a simple fluid such as argon. With the methods presented here, numerical integration and differentiation are not necessary for the square-well and Yukawa fluids. Numerical integration cannot be easily avoided in the case of the Lennard-Jones fluid. However, numerical differentiation with respect to the density is not required.
\end{abstract}

Key words: perturbation theory, inverse temperature expansion, compressibility approximations, analytic methods

PACS: $02.30 . Q y, 02.30 . R z, 05.20 . J j, 05.70 . C e, 64.30 .+t$

\section{Introduction}

Only a venerable scientist, such as the author, can recall the times when the theory of a dense fluid was considered to be an intractable problem. This unsatisfactory situation changed in the last half of the previous century first through the availability of computer simulations [1] of the properties of a fluid that gave unambiguous results for their thermodynamic (and even transport) properties, followed by the development of perturbation theory [2 -4] and some integral equations, for example, the Percus-Yevick (PY) [5] and the mean spherical (MSA) [6] approximations. Of course, there has been a great deal of additional progress during the past forty years. However, this paper will deal with the early work.

The thermodynamic properties and correlation functions of a simple fluid result from the pair interactions, $u\left(R_{i j}\right)$, between pairs of particles $(i$ and $j)$, where $R_{i j}=\left|\mathbf{r}_{i}-\mathbf{r}_{j}\right|$ and $\mathbf{r}_{i}$ is the position of the center of particle $i$. For simplicity, the further assumption that the forces are central is made. This discussion is further limited to one component fluids.

In its simplest form, the idea in perturbation theory is to divide the pair potential into two parts,

$$
u(R)=u_{0}(R)+u_{1}(R),
$$

where $u_{0}(R)$ is the pair interaction of the unperturbed system (usually the hard sphere or HS fluid), whose thermodynamic properties and correlation functions are well-known, preferably through accurate analytic functions or accurate tabulations, and $u_{1}(R)$ is the perturbation potential. The Helmholtz free energy, which is simply related to the partition function, is then expanded in a series. Less frequently, the pair correlation function is also expanded in a series. For perturbation theory to be successful, the perturbation must be small, in some sense, so that the series converges

\footnotetext{
*E-mail: doug@chem.byu.edu
} 
rapidly and one or two perturbation terms are sufficient to give good results. One reason why the HS fluid is so useful in perturbation theory is that the thermodynamic properties and correlation functions of this fluid, that are functions of the density but not the temperature, are given accurately by the Percus-Yevick (PY) theory. Interestingly, the PY and MSA theories become identical for the HS fluid. They differ for other fluids. Generally speaking the PY is not very accurate for fluids other than the HS fluid. Perhaps it would be preferable to refer to the PY HS results as MSA results. However, for historical reasons the HS results are called PY results.

Thus, the most frequent choice for the unperturbed system is the HS potential, for which

$$
u_{0}(R)= \begin{cases}\infty, & R<\sigma \\ 0, & R>\sigma\end{cases}
$$

where the quantity $\sigma$ is the diameter of the (reference) hard sphere system. Often the hard sphere diameter is represented by the symbol $d$. The HS fluid is useful in perturbation theory because the thermodynamic properties and correlation functions are given accurately by analytic expressions when the PY theory is employed and the HS correlation functions are a good approximation to the correlation functions of the fluid of interest.

Here we will discuss perturbation theory for three cases, where $u_{1}(R)$ is given firstly by the square-well fluid, where

$$
u_{1}(R)= \begin{cases}-\epsilon, & \sigma<R<\lambda \sigma \\ 0, & R>\lambda \sigma\end{cases}
$$

secondly, by the Yukawa fluid, where

$$
u_{1}(R)=-\frac{\epsilon \sigma}{R} \exp \left[-\frac{z}{\sigma}(R-\sigma)\right]
$$

for $R>\sigma$, and, thirdly, by the Lennard-Jones (LJ) 12-6 fluid, where

$$
u(R)=4 \epsilon\left(\left\{\frac{\sigma}{R}\right\}^{12}-\left\{\frac{\sigma}{R}\right\}^{6}\right)
$$

The LJ potential gives the properties of a simple fluid, such as argon, with reasonably good accuracy. The values, $\lambda=1.5$ and $z=1.8$ are used conventionally for the square-well and Yukawa fluids, respectively. With these values, the square-well and Yukawa fluids mimic the LJ fluid, and thus argon, with similar values of $\epsilon$ and $\sigma$.

The division of the LJ potential into $u_{0}(R)$ and $u_{1}(R)$ is less clear because the repulsive part of this potential is not infinitely steep. However, Barker and Henderson(BH) [3] showed that $u_{1}(R)$ can be taken to be the portion of the LJ potential for the region, $R>\sigma$, for which the LJ potential is negative. Thus, $u_{0}(R)$ is the portion of the LJ potential for the region, $R<\sigma$, for which the LJ potential is positive. This is not a very convenient reference fluid since its direct use would require a knowledge of this fluid for every temperature and density. However, BH showed that this reference fluid could be replaced, with good accuracy, with a HS fluid whose diameter $d$ was given by

$$
d=\int_{0}^{\sigma}[1-\exp \{-\beta u(R)\}] \mathrm{d} R,
$$

where $\beta=1 / k T$ with $k$ and $T$ being the Boltzmann constant and temperature, respectively. This effective diameter, $d$, is slightly smaller than $\sigma$ and is a weak function of $T$ and independent of the density. The effective diameter can be obtained at the beginning of a calculation and fit to some function. Then a value of $d$ can be determined from this fitted function or interpolated from a table. The $\mathrm{BH}$ procedure makes the application of perturbation theory to the LJ fluid almost as simple as for the other fluids. 


\section{Perturbation theory}

The idea of perturbation theory is that the Helmholtz free energy, $A$, the pressure, $p$, or any thermodynamic property can be expanded in a power series in $\epsilon$. Ultimately, this becomes a power series in $\beta \epsilon$. Less frequently, the radial distribution function, $g(R)$ is also expanded in a power series in this same parameter. At high densities, this series is rapidly convergent even though $\beta \epsilon$ is as large as 1.4 near the triple point. The convergence at high densities is due to the fact that the higher order terms are fluctuations and these fluctuations are inhibited at high densities because the hard (or nearly hard) cores of the molecules are pushed up against each other, either by external pressure or the attractive forces between the molecules (often called an internal pressure).

Thus,

$$
\frac{A}{N k T}=\frac{A_{0}}{N k T}+\sum_{n=1}^{\infty}(\beta \epsilon)^{n} \frac{A_{n}}{N k T},
$$

where $N$ is the number of molecules in the system. The first term is the Helmholtz function for the hard sphere reference system and is given by

$$
\frac{A_{0}}{N k T}=\frac{3}{2} \ln \Lambda-1-\ln \rho+\frac{4-3 \eta}{(1-\eta)^{2}}
$$

with $\Lambda^{2}=h^{2} / 2 \pi m k T, h$ being Planck's constant, $m$ the molecular mass, $\eta=\pi \rho d^{3} / 6, \rho=N / V$, $V$ is the volume of the system, and $d$ is equal to $\sigma$ for the square-well and Yukawa potentials and the value given by equation (6) in the case of the LJ potential. There is no inconsistency. Equation (6) yields $d=\sigma$ for the square-well and Yukawa potentials, or any potential with a hard core. Equation (8) results from, and is consistent with, the well-known Carnahan-Starling equation,

$$
\frac{p_{0} V}{N k T}=\frac{1+\eta+\eta^{2}-\eta^{3}}{(1-\eta)^{3}},
$$

where $p_{0}$ is the pressure of the reference system.

The first order term, $A_{1}$ is given by

$$
\frac{A_{1}}{N k T}=2 I_{1}
$$

where

$$
I_{1}=\pi \rho \int_{\sigma}^{\infty} R^{2} u_{1}^{*}(R) g_{0}(R) \mathrm{d} R,
$$

with $u_{1}^{*}(R)=u_{1}(R) / \epsilon$ and $g_{0}(R)$ is the radial distribution function, of the reference fluid. The resulting expression for the first order term, $p_{1}$, in an analogous expansion for the pressure, $p$, of the fluid is

$$
\frac{p_{1} V}{N k T}=2 \eta \frac{\partial I_{1}}{\partial \eta}
$$

A rigorous, but not very useful, expression for $A_{2}$ involves three and four particle correlation functions. $\mathrm{BH}$ have calculated $A_{2}$ from these rigorous expressions but with limited success due to the fact that the final result is a small difference between much larger values. Because of this problem, two approximations [2], also due to $\mathrm{BH}$, are of interest. One is the macroscopic compressiblity approximation and the other is the microscopic compressiblity approximation. Both approximations derive their name and form from the fact that the second order term is, in a general sense, a density fluctuation and fluctuations in a bulk system are related to the compressiblity. The approximations of $\mathrm{BH}$ are fairly accurate and appealing since fluctuations are related to the compressiblity. 


\section{Macroscopic approximation}

At low densities, one would expect $A_{2}$ to be given by an expression similar to equation (10) but involving $\left[u^{*}(R)\right]^{2}$. It is plausible that the fluctuation term, $A_{2}$ might be such an integral but with a prefactor of $k T(\partial \rho / \partial p)_{0}$, which is the compressiblity of the reference HS fluid. This approximation is the macroscopic compressiblity (mac) approximation. Thus,

$$
\frac{A_{2}}{N k T}=-\frac{(1-\eta)^{4}}{(1+2 \eta)^{2}} I_{2}
$$

where

$$
I_{2}=\pi \rho \int_{\sigma}^{\infty} R^{2}\left[u^{*}(R)\right]^{2} g_{0}(R) \mathrm{d} R
$$

The PY expression for the HS fluid compressiblity has been used. This is a little less accurate than the Carnahan-Starling expression but, given the fact that the macroscopic approximation is itself an approximation, the use of a slightly more complex result does not seem warranted. The corresponding expression for the pressure is

$$
\frac{p_{2} V}{N k T}=4 \eta \frac{(1-\eta)^{3}(2+\eta)}{(1+2 \eta)^{3}} I_{2}-\eta \frac{(1-\eta)^{4}}{(1+2 \eta)^{2}} \frac{\partial I_{2}}{\partial \eta}
$$

\section{Microscopic approximation}

BH suggested further that since $A_{2}$ was a fluctuation and this was a microscopic fluctuation, it would be more attractive to use what they called the microscopic compressiblity (mic) approximation, $\partial\left[\rho g_{0}(R)\right] / \partial p$, instead of $[\partial \rho / \partial p] g_{0}(R)$. Using

$$
\left(k T \frac{\partial}{\partial p}\right)_{0}=\left(k T \frac{\partial \rho}{\partial p} \frac{\partial}{\partial \rho}\right)_{0}=\frac{(1-\eta)^{4}}{(1+2 \eta)^{2}} \frac{\partial}{\partial \rho}
$$

this yields

$$
\frac{A_{2}}{N k T}=-\eta \frac{(1-\eta)^{4}}{(1+2 \eta)^{2}} \frac{\partial I_{2}}{\partial \eta} .
$$

Again the PY expression for the HS compressiblity has been used. Differentiating once more yields

$$
\frac{p_{2} V}{N k T}=\eta \frac{(1-\eta)^{3}\left(1-7 \eta-6 \eta^{2}\right)}{(1+2 \eta)^{3}} \frac{\partial I_{2}}{\partial \eta}-\eta^{2} \frac{(1-\eta)^{4}}{(1+2 \eta)^{2}} \frac{\partial^{2} I_{2}}{\partial \eta^{2}}
$$

The mic approximation is mentioned mainly for historical reasons. For those fluids to which they have been applied, the mac and mic approximations give very similar results. It is probably satisfactory to use the mac approximation and avoid an extra differentiation. This will be done here and the mac approximation will be referred to simply as the compressiblity approximation (CA). An extension of the CA to higher order terms has been given by Praestgaard and Toxvaerd [7]

\section{Integrals}

The task is to calculate $I_{1}$ and its density derivative and $I_{2}$ and its first and second derivatives. The function $g_{0}(R)$ is given quite accurately from the PY theory; its Laplace transform was obtained exactly by Wertheim [8, 9]. An exact expression for the PY $g_{0}(R)$ is not available but an exact analytic result for the PY $g_{0}(R)$ for $d<R<5 d$ has been found by Smith and Henderson [10] and for $d<R<6 d$ by Henderson and Smith [11]. A computer code that gives these results can be found in the supplementary material of Smith et al. [12]. A straightforward procedure would be to obtain $I_{1}$ and $I_{2}$ by numerical integration of the Smith-Henderson results and then obtain 
the derivatives numerically with a probable compounding of numerical errors. However, simpler methods are available and these will now be considered. They differ somewhat for the square-well, Yukawa, and LJ potentials and, as a result, these three potentials are considered separately.

Before doing so, it is useful to define the Wertheim polynomials [9],

$$
L(s)=(1+\eta / 2) s+1+2 \eta
$$

and

$$
S(s)=(1-\eta)^{2} s^{3}+6 \eta(1-\eta) s^{2}+18 \eta^{2} s-12 \eta(1+2 \eta) .
$$

Also, we will need the density derivatives of these polynomials. They are

$$
\begin{gathered}
\frac{\partial L(s)}{\partial \eta}=L^{\prime}(s)=\frac{s}{2}+2, \\
\frac{\partial S(s)}{\partial \eta}=S^{\prime}(s)=-2(1-\eta) s^{3}+6(1-2 \eta) s^{2}+36 \eta s-12(1+4 \eta),
\end{gathered}
$$

and

$$
\frac{\partial^{2} S(s)}{\partial \eta^{2}}=S^{\prime \prime}(s)=2 s^{3}-12 s^{2}+36 s-48 .
$$

The higher order density derivatives are zero. For a simple notation, these derivatives will be represented by $L^{\prime}(s)$, etc. It should be kept in mind that $L^{\prime}(s)$, etc, are derivatives with respect to $\eta$, not $s$.

\section{Square-well potential}

In principle, the integrals $I_{n}$ could be obtained for any value of $\lambda$ by integrating the analytical expressions for $g_{0}(R)$ that were obtained by Smith and Henderson [10, 11]. In general, this would be impractical because these expressions become rather complicated if $\lambda>2$. However, for $d<R<2 d$, the result is fairly simple,

$$
x g_{0}(x)=\sum_{i=1}^{3} c_{i} \exp \left(m_{i} x\right)
$$

where $x=R / d$ ( $d=\sigma$ for the square-well potential) and the $m_{i}$ are the three roots of the cubic equation

$$
S\left(m_{i}\right)=0 .
$$

The $m_{i}$ are given by analytic expressions but two are complex (i.e, have an imaginary part) and the calculation is best done using complex arithmetic. The $c_{i}$ and $m_{i}$ can be obtained from the subroutine GRPRLM of the FORTRAN code of Smith and Henderson that is given in the supplementary material of the recent paper of Smith et al. [12].

As long as $\lambda<2$, these simple expressions are all that is needed. Using the fact that $u_{1}^{*}(R)$ is either zero or $-1, I_{n}=(-1)^{n-1} I_{1}$ and, hence, only $I_{1}$ need be determined. The result for $I_{1}$ is

$$
I_{1}=-6 \eta \sum_{i=1}^{3} \frac{c_{i} J_{i}}{m_{i}^{2}}
$$

where

$$
J_{i}=\left(\lambda m_{i}-1\right) \exp \left(\lambda m_{i}\right)-\left(m_{i}-1\right) \exp \left(m_{i}\right) .
$$

The density derivatives $I_{1}^{\prime}$ and $I_{1}^{\prime \prime}$ can be obtained by straightforward, but tedious, differentiation. For example,

$$
I_{1}^{\prime}=-6 \sum_{i=1}^{3} \frac{c_{i} J_{i}}{m_{i}^{2}}-6 \eta \sum_{i=1}^{3} \frac{J_{i}}{m_{i}^{2}} \frac{\partial c_{i}}{\partial \eta}+12 \eta \sum_{i=1}^{3} \frac{c_{i} J_{i}}{m_{i}^{3}} \frac{\partial m_{i}}{\partial \eta}-6 \eta \sum_{i=1}^{3} \frac{c_{i}}{m_{i}^{2}} \frac{\partial J_{i}}{\partial \eta}
$$


and

$$
\frac{\partial J_{i}}{\partial \eta}=m_{i}\left[\lambda^{2} \exp \left(\lambda m_{i}\right)-\exp \left(m_{i}\right)\right] \frac{\partial m_{i}}{\partial \eta} .
$$

The derivative of $m_{i}$ is obtained by differentiating equation (25) to get

$$
\frac{\partial m_{i}}{\partial \eta}=\frac{\partial S\left(m_{i}\right)}{\partial \eta}\left(\frac{\partial S\left(m_{i}\right)}{\partial m_{i}}\right)^{-1} .
$$

An alternative procedure would be to use a result of Barker and Henderson [4] who have fit $I_{1}$ for the square-well potential $(\lambda=1.5)$. Their simple result is

$$
I_{1}=C\left[1-\exp \left(-\frac{1.5 \rho^{*}}{\sqrt{2}-\rho^{*}}\right)-\frac{1.5 \rho^{*}}{\sqrt{2}}\right]+P \rho^{*}+Q \rho^{* 2},
$$

where $C=-4.230411, P=-2.487096$, and $Q=-1.213608$, and $\rho^{*}=\rho \sigma^{3}$. This fit of $I_{1}$ was made using the simulation results for $g_{0}(x)$. However, the difference between the PY and simulation results for $g_{0}(x)$ is not significant. This result is easily differentiated.

\section{Yukawa potential}

The Yukawa potential is the easiest of the three potentials because $I_{1}$ is directly related to the Laplace transform of the PY $g_{0}(x)$ that has been obtained as an analytic expression by Wertheim [9]. The result for $I_{1}$ is

$$
I_{1}=-6 z \exp (z) \frac{\eta L(z)}{12 \eta L(z)+S(z) \exp (z)} .
$$

The density derivative is obtained easily.

$$
I_{1}^{\prime}=-6 z \exp (2 z) \frac{L(z) S(z)+\eta\left[L^{\prime}(z) S(z)-L(z) S^{\prime}(z)\right]}{[12 \eta L(z)+S(z) \exp (z)]^{2}} .
$$

This may be continued to obtain the higher order derivatives.

Additionally, the integral $I_{2}$ need be calculated. This is not so straightforward. It could be calculated numerically but this is unappealing. Since the compressiblity approximation is an approximation, a good estimate to $I_{2}$ could be obtained by renormalizing $I_{1}$ so that it is equal to $I_{2}$ at low densities. The result is

$$
I_{2}=-\frac{z}{2(z+1)} I_{1}
$$

Since $I_{1}$ is negative, $I_{2}$ is positive. This the version of the compressiblity approximation that will be used here for the Yukawa fluid. Equation (34) is accurate at low densities but is an underestimation of the magnitude of $I_{2}$ at high densities. However, this is not so important since the density dependence of $I_{2}$ is determined mainly by the compressiblity prefactor that is small at high densities and suppresses $I_{2}$.

\section{Lennard-Jones potential}

The application of perturbation theory to the LJ potential is complicated by the fact that $d<\sigma$. The value of the first order term, $A_{1}$, is determined by the integral from $\sigma$ to $\infty$. However, we may write

$$
\int_{\sigma}^{\infty} R^{2} u^{*}(R) g_{0}(R) \mathrm{d} R=\int_{d}^{\infty} R^{2} u^{*}(R) g_{0}(R) \mathrm{d} R-\int_{d}^{\sigma} R^{2} u^{*}(R) g_{0}(R) \mathrm{d} R .
$$


One procedure would be to integrate numerically the integral on the left hand side of the above equation and differentiate this result numerically or fit the value of the integral to a function and differentiate this fitted function. Of course, analytic integration, using the Smith-Henderson formulae, is, in principle, possible but this would involve repeated integrations by parts.

Although numerical integration of the more important first integral cannot be avoided easily, a method that avoids numerical differentiation with respect to the density will be outlined. Using equation (52) of the earlier paper in this series [14], it follows that

$$
\int_{d}^{\sigma} R^{2} u^{*}(R) g_{0}(R) \mathrm{d} R=4 d^{3} \int_{0}^{\infty}\left[\left(\frac{\sigma}{d}\right)^{12} \frac{s^{10}}{10 !}-\left(\frac{\sigma}{d}\right)^{6} \frac{s^{4}}{4 !}\right] G_{0}(s) \mathrm{d} s
$$

where

$$
G_{0}(s)=\frac{s L(s)}{12 \eta L(s)+\exp (s) S(s)}
$$

is the Laplace transform of $g_{0}(R)$ that is obtained from the PY theory. Since numerical integration cannot be avoided easily, equation (36) by itself would seem not to have much advantage over an integration in direct space. However, it does have the advantage that $G_{0}(s)$ is known for all $s$ whereas $g_{0}(R)$ is known analytically only for $R<6 d$. Further, equation (36) can be differentiated directly without numerical differentiation. All that is needed is the density derivative of $G_{0}(s)$. The result is

$$
\frac{\partial G_{0}(s)}{\partial \eta}=s \frac{-12 L^{2}(s)+\left[L^{\prime}(s) S(s)-L(s) S^{\prime}(s)\right] \exp (s)}{[12 \eta L(s)+\exp (s) S(s)]^{2}} .
$$

Equation (38) is perfectly satisfactory. However, the elegant result of Frisch et al. [14] is worth noting,

$$
\frac{\partial\left[\eta G_{0}(s)\right]}{\partial \eta}=s^{4} \exp (s)(1+2 \eta) \frac{1+2 \eta+(1-\eta) s}{[12 \eta L(s)+\exp (s) S(s)]^{2}} .
$$

Higher order density derivatives can be obtained easily.

In principle, the second integral could be annoying. However, at the temperatures for which the LJ fluid is a liquid (or a near liquid), $d$ is only slightly less than $\sigma$. As a result, the trapizoid rule may be used. Thus,

$$
\int_{d}^{\sigma} R^{2} u^{*}(R) g_{0}(R) \mathrm{d} R=\frac{d^{2}}{2} u^{*}(d) g_{0}(d)(d-\sigma)
$$

with $g_{0}(d)=(1+\eta / 2) /(1-\eta)^{2}$ from the PY theory. Equation (40) makes use of the fact that $u(\sigma)=0$. Because $d$ is independent of the density, the density derivatives of equation (40) are obtained straightforwardly. The integral $I_{2}$ and its density derivative can be obtained by analogous methods.

Bear in mind that because $d$ is a function of $T$, the $A_{n} / N k T$ for the LJ potential are (weak) functions of $T$. Hence, the values of $A_{n} / N k T$ should be computed at each temperature.

\section{Some typical results}

Since the square-well potential with $\lambda=1.5$, the Yukawa potential with $z=1.8$ and the LJ potential are all meant to yield the thermodynamic properties of a simple fluid such as argon, the results obtained using these potentials are similar. There is no reason to consider all three fluids; the Yukawa fluid will be used here.

Results for $A_{1} / N k T$ and $A_{2} / N k T$ are plotted in figures 1 and 2. Two facts emerge from figure1. Firstly, $A_{1} / N k T$ is approximately a linear function of $\rho$. Even though $g_{0}(R)$ is a strong function of $\rho$, its integral is not. The assumption that $A_{1} / N k T$ is a linear function of $\rho$ is one of the assumptions of the van der Waals theory. Secondly, there is no significant difference if the PY or the simulation results are used to calculate $A_{1} / N k T$ from $g_{0}(R)$. 


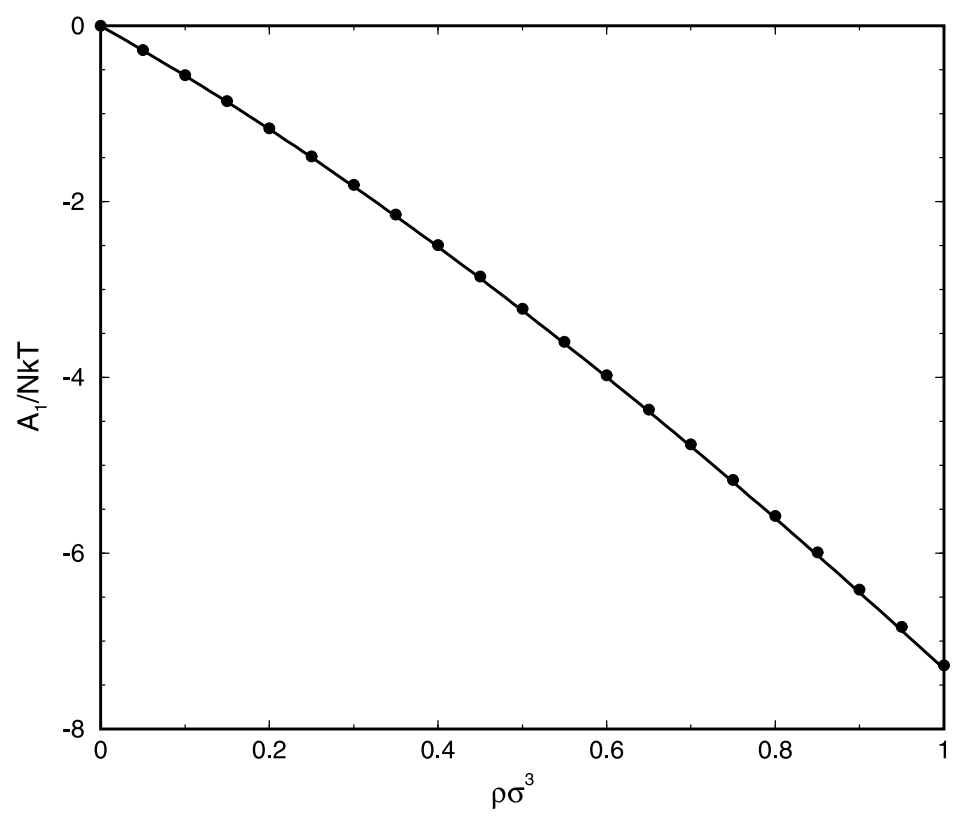

Figure 1. First order perturbation term for a Yukawa fluid. The points give the simulation results and the curve is calculated from the PY $g_{0}(R)$ using equation (32).

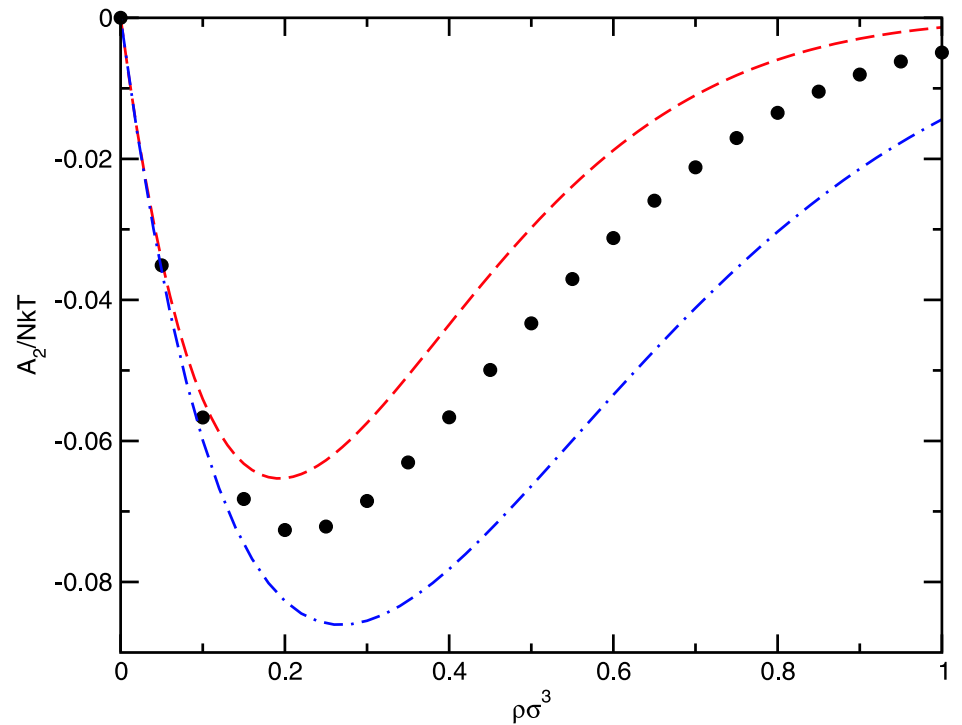

Figure 2. Second order perturbation term for the Yukawa fluid. The points give the simulation results, the dashed curve gives the MSA results, and dot dash curve gives the CA results.

Results for $A_{2} / N k T$ are plotted in figure 2, where exact results, obtained from simulations, are compared with the MSA and the CA results. The second order term, $A_{2} / N k T$, is small compared with the first order term, $A_{1} / N k T$, indicating rapid convergence of the perturbation theory. The higher order $A_{n} / N k T$ would be even smaller. Only in the immediate vicinity of the critical density, do the higher order terms contribute significantly. We will not concern ourselves with this point since perturbation theory cannot be expected to be a satisfactory theory of the critical point because an infinite number of terms in the series would be required. The convergence is particularly rapid at high densities, where $A_{2} / N k T$ becomes small, and fluctuations are suppressed because the fluid and reference fluid are nearly incompressible. Further, it is to be noted that there is little difference 
between the MSA and perturbation theory approximations for $A_{2} / N k T$. They are alike and close to the exact results. The MSA results are slightly better but the CA results are more easily obtained. The CA results are a little too large in magnitude (more negative) but this compensates in part for the neglect of the higher order terms. If the results of the CA, based on a calculation of $I_{2}$ rather than equation (14), were used, the results would be somewhat larger in magnitude (more negative) but would still be a good approximation to the simulation results.

Results for the thermodynamics properties of these fluids are not reported since they have been given for the square-well and LJ fluids by Barker and Henderson [2 4]. Henderson et al. [15] have reported the thermodynamic properties of the Yukawa fluid using the MSA. The perturbation theory results are almost identical.

Weeks, Chandler, and Andersen(WCA) [16] have formulated a somewhat different version of perturbation theory. It is different only for pair potentials with a soft core, such as the LJ potential. There is no difference between the two versions of perturbation theory for the square-well and Yukawa fluids. The WCA theory converges somewhat faster for the LJ fluid However, the BH theory with the CA second order term is equally user friendly and accurate.

\section{Summary}

The purpose of this paper is to report some methods other than straightforward numerical integration and differentiation that yield the integrals that arise from perturbation theory and to draw attention to the compressiblity approximations of Barker and Henderson. After their initial work Barker and Henderson attempted to compute the integrals that contribute to $A_{2} / N k T$ using simulations or higher order correlation functions. In retrospect, this was a nobel effort but probably a mistake. It is difficult to obtain $A_{2} / N k T$ with sufficient accuracy by such methods because this second order term results from extensive cancellation between much larger quantities. The simpler $\mathrm{CA}$ is recommended. It is easier to use and, for simple liquids, is of comparable accuracy to such approximations as the MSA.

In closing, it is worth noting that the CA gives some intuitive insight into the singular behavior of the thermodynamic functions at the critical point. In principle, the compressiblity of the fluid might be used in the CA instead of the compressiblity of the reference hard sphere fluid. Since $\partial p / \partial \rho$ vanishes at the critical point, a singularity would result. It is likely that this argument is too qualitative to produce the correct behavior of the thermodynamic properties at the critical point but it does have some appeal.

\section{References}

1. Hansen J.-P., McDonald I.R., Theory of Simple Liquids, 3rd. Edition. Academic Press, London, UK, 2006.

2. Barker J.A., Henderson D., J. Chem. Phys., 1967, 47, 2856.

3. Barker J.A., Henderson D., J. Chem. Phys., 1967, 47, 4714.

4. Barker J.A., Henderson D., Rev. Mod. Phys., 1976, 48, 587.

5. Percus J.K., Yevick G.J., Phys. Rev., 1958, 110, 1.

6. Lebowitz J.L., Percus J.K., Phys. Rev., 1966, 144, 251.

7. Praestgaard E., Toxvaerd S., J. Chem. Phys., 1970, 53, 2389.

8. Wertheim M., Phys. Rev. Lett., 1963, 10, 321.

9. Wertheim M., J. Math. Phys., 1964, 5, 643.

10. Smith W.R., Henderson D., Mol. Phys., 1970, 19, 411.

11. Henderson D., Smith W.R., J. Stat. Phys., 1978, 19, 191.

12. Smith W.R., Henderson D., Leonard P.J., Barker J.A., Grundke E.W., Mol. Phys., 2008, 106, 3.

13. Henderson D., Cond. Matter Phys., 2009, 12, 127.

14. Frisch H.L., Katz J.L., Praestgaard E., Lebowitz J.L., J. Phys. Chem., 1966, 70, 2016.

15. Henderson D., Waisman E., Lebowitz J.L., Blum L., Mol. Phys., 1978, 35, 241.

16. Weeks J.D., Chandler D., Andersen H.C., J. Chem. Phys., 1971, 54, 5237. 


\title{
Зручні формули для деяких інтегралів у теорії збурень
}

\author{
Д. Гендерсон \\ Факультет хімії і біології, Університет Брайхем Янг, Прово, США
}

\begin{abstract}
Вирази для вільної енергії і тиску плину, отримані за допомогою теорії збурень, включають в себе інтеграли як від кореляційних функцій твердих сфер, так і від їхніх похідних за густиною. В більшості застосувань ці інтеграли можуть бути отримані, також і чисельно, із застосуванням простої процедури. що використовує формули і комп'ютерні коди для кореляційних функцій твердих сфер, які були отримані раніше [Mol. Phys., 2007, 106, 2; Condens. Matter Phys., 2009, 12, 127 ], а також чисельне диференціювання за густиною, що може привести при цьому до можливих похибок. В цій статті, яка є другою із запланованої серії трьох статей, що представляють записки лекцій автора, пропонуються складніші методи. Розглядаються відомі моделі плину, взаємодія в яких представляється за допомогою трьох потенціалів, а саме: потенціалу типу прямокутної ями, потенціалу Юкави і потенціалу Леннарда-Джонса. Кожний з цих модельних плинів широко використовується у теоретичних та інженерних обчисленнях для опису такого простого плину як аргон. 3 використанням методів, представлених у цій статті, чисельне інтегрування і диференціювання перестає бути необхідним у випадках потенціалу типу прямокутної ями і потенціалу Юкави. Чисельного інтегрування не можна легко уникнути у випадку потенціалу Леннарда-Джонса. Проте, чисельне диференціювання за густиною не вимагається.
\end{abstract}

Ключові слова: теорія збурень, розклад за степенями оберненої температури, наближення стисливості, аналітичні методи 